\begin{tabular}{|c|l|}
\hline Title & $\begin{array}{l}\text { Structural Characteristics in a Stable Metallic ET Salt with Unusually High Oxidation State (ET: } \\
\text { Bis(ethylenedithio)tetrathiafulval ene) }\end{array}$ \\
\hline Author(s) & Minemawari, Hiromi; Jose, Judy Fe F.; Takahashi, Yukihiro; Naito, Toshio; Inabe, Tamotsu \\
\hline Citation & $\begin{array}{l}\text { Bulletin of the Chemical Society of Japan, 85(3), 335-340 } \\
\text { https://doi.org/40.1246/0csj.20110253 }\end{array}$ \\
\hline Issue Date & 2012-03 \\
\hline Doc URL & http://hdl.handle.net/2115/52228 \\
\hline Type & article (author version) \\
\hline File Information & BCSJ85-3_335-340.pdf \\
\hline
\end{tabular}

Instructions for use 


\title{
Structural Characteristics in a Stable Metallic ET Salt with Unusually High Oxidation State (ET = bis(ethylenedithio)tetrathiafulvalene)
}

Hiromi Minemawari, ${ }^{\dagger}$ Judy Fe F. Jose, Yukihiro Takahashi, Toshio Naito, ${ }^{\#}$ and Tamotsu Inabe*

Department of Chemistry, Graduate School of Science, Hokkaido University, Sapporo 060-0810, Japan.

Received August 23, 2011; E-mail: inabe @sci.hokudai.ac.jp

\begin{abstract}
:
Electrolysis of ET in a mixed solvent of methanol/ $\mathrm{CHCl}_{3}$ with bromide yielded $(\mathrm{ET})_{3}\left(\mathrm{Br}_{3}\right)_{5}$, in which an unusually high formal charge of $\mathrm{ET}^{5 / 3+}$ is achieved. ET forms a two-dimensional conducting sheet incorporating $\mathrm{Br}_{3}{ }^{-}$. Despite the high formal charge of the ET cations, the strong electrostatic attractions between the ET cations and $\mathrm{Br}_{3}{ }^{-}$ support aggregation of the ET cations, allowing sufficient $\pi-\pi$ overlap for delocalization of the $\pi$-electrons. The crystal shows stable metallic behavior down to low temperatures, being consistent with the metallic band structure with two-dimensional Fermi surfaces and Pauli-like paramagnetism down to low temperatures. The lack of any indication of instability in the metallic phase due to correlation effects is considered to result from an ionic-crystal-like structure, in which the lattice is more resistant toward lattice deformation and/or shrinkage than in typical partially oxidized salts with low formal charges.
\end{abstract}

${ }^{\dagger}$ Present address: AIST, Tsukuba 305-8562, Japan

"Present address: Graduate School of Science and Engineering, Ehime University, Matsuyama 790-8577, Japan 
Since the 1970s, an enormous number of organic molecular conductors have been developed. In general, the formal charge of them does not exceed +1 (or -1 ). When the formal charge becomes exactly one, charge transport becomes difficult because of the extra energy required to overcome the Coulomb repulsion when a molecule accommodates two charges. On the other hand, when the formal charge exceeds +1 (or -1 ), band filling becomes below (or above) the half-filled level. Thus, the nature of the carriers may change from holes to electrons (or electrons to holes) as the formal charge goes beyond 1. In this situation, electrostatic repulsion between the charge carriers is considered to be identical to that in partially oxidized (reduced) salts with formal charges not exceeding one. However, electrostatic repulsion between the molecules becomes much larger, since each molecule has a charge exceeding +1 (or -1 ). Therefore, it becomes much more difficult for molecules to pack tightly enough to gain sufficient $\pi-\pi$ interaction in this situation.

Metallic conductors with formal charges exceeding one have not been extensively reported. One such example is a salt composed of TTM-TTP (2,5-bis[4,5-bis(methylthio)-1,3-dithiol-2-ylidene]-1,3,4,6-tetrathiapentalene); the formal charge of TTM-TTP in (TTM-TTP) $\left(\mathrm{I}_{3}\right)_{5 / 3}$ is $+5 / 3 .{ }^{1}$ This molecule contains two conjugated TTF (tetrathiafulvalene) frameworks, each of which shows two-step oxidation. TTM-TTP thus shows reversible four-step oxidation up to [TTM-TTP $]^{4+}$, and the high oxidation states are relatively stable compared with ordinary donor molecules. ${ }^{2}$ The $\pi$-conjugation is also more extended than those in ordinary donors, and this makes the overlap integral (proportional to the transfer integral) so large that it overcomes the electrostatic repulsion between the molecules with charges of the same sign. The delocalization energy of the charges is large enough to diminish the on-site Coulomb repulsion energy, and the salt with a formal charge of +1 also shows high conductivity. ${ }^{3}$

The other type of metallic conductors can be found in salts composed of $\mathrm{C}_{60}$. In $\mathrm{A}_{3} \mathrm{C}_{60}\left(\mathrm{~A}=\right.$ alkaline metal ion) $\mathrm{C}_{60}$ has a very high formal charge of -3 , but the resulting salt shows metallic conduction and superconductivity. ${ }^{4}$ The last group is composed of salts with ET. In $(\mathrm{ET})_{6}\left(\mathrm{AuBr}_{2}\right)_{6} \mathrm{Br}$ (TIE) $(\mathrm{TIE}=$ tetraiodoethylene; metallic down to 50 $\mathrm{K}$ ), the formal charge of ET is $+7 / 6 .{ }^{5}$ In $(\mathrm{ET})_{2} \mathrm{Cu}_{3} \mathrm{Br}_{7}\left(\mathrm{H}_{2} \mathrm{O}\right)$ (metallic down to $50 \mathrm{~K}$ ) in which ET functions as a ligand of $\mathrm{Cu}^{+}$ion, the formal charge of ET was reported to be $+2 .{ }^{6}$ We have recently found another example of $(\mathrm{ET})_{3}\left(\mathrm{Br}_{3}\right)_{5}$, in which the formal charge of $\mathrm{ET}$ is $+5 / 3$. $^{7}$ 
In our previous report, ${ }^{7}$ the crystal structure and transport properties of $(\mathrm{ET})_{3}\left(\mathrm{Br}_{3}\right)_{5}$ were briefly described. Band calculation showed a metallic feature, and the transport was indeed metallic at high temperatures. However, the resistivity showed a gradual increase below $120 \mathrm{~K}$, while the magnetic susceptibility revealed Pauli-like paramagnetism down to $2 \mathrm{~K}$. In order to clarify this inconsistency, we have re-measured the electrical conductivity, and observed its metallic behavior down to low temperature. In this paper, we describe the crystal growth, detailed structural features, and physical properties of $(\mathrm{ET})_{3}\left(\mathrm{Br}_{3}\right)_{5}$ and discuss the structural characteristics of the metallic conductors with formal charges exceeding one.

\section{Experimental}

Materials. Single crystals of $(\mathrm{ET})_{3}\left(\mathrm{Br}_{3}\right)_{5}$ were obtained by the same method reported. $^{7}$ Galvanostatic oxidation was performed using an electrocrystallization cell equipped with a glass frit between two compartments. Brownish-black platelet crystals were grown on the anode at a constant current of 4.0-7.0 $\mu \mathrm{A}$ for a week applied to a solution containing ca. $15 \mathrm{mg}$ of ET and ca. $50 \mathrm{mg}$ of TPP $\mathrm{Br}$ or PTMA Br (TPP = tetraphenylphosphonium and PTMA = trimethylphenylammonium). The solvent system was a mixture of methanol and chloroform (typically $10 \mathrm{ml}$ and $20 \mathrm{ml}$, respectively). Elemental analyses of the bulk product indicated slightly larger $\mathrm{C}$ and $\mathrm{H}$ contents (Calcd: C, 15.32; H, 1.03, S, 32.71\%. Found: C, 16.24; H, 1.24; S, 32.82\%), indicating that a small amount of ET salts with lower bromine contents coexisted. However, thin platelets with well-developed flat planes always gave the same cell parameters which could be assigned to $(\mathrm{ET})_{3}\left(\mathrm{Br}_{3}\right)_{5}$. The byproduct was crystallized in a form of aggregation of micro-crystals of which identification was not performed due to its small quantity and poor crystallinity. For the measurements described below, thin platelets characteristic of $(\mathrm{ET})_{3}\left(\mathrm{Br}_{3}\right)_{5}$ were selected.

Measurements. The temperature dependence of the lattice constants was measured with a Rigaku R-AXIS Rapid imaging plate diffractometer with graphite-monochromated Mo $K \alpha$ radiation. The temperature was regulated by a cold nitrogen gas flow in the range of 130-280 K. Lattice parameters were obtained using $889(280 \mathrm{~K})-1315(130 \mathrm{~K})$ independent reflections with a least-squares refinement. 
Single-crystal resistivity measurements were carried out using a four-probe method in the temperature range of 5-300 K. Gold thin layers (about $100 \mathrm{~nm}$ thick) were deposited on the crystal surface as contacts between the crystal and gold lead wires. The temperature dependence was measured using a closed cycle cryostat system in the temperature range of 5-300 K. The sample chamber was evacuated for about $10 \mathrm{~min}$. at room temperature, and kept in vacuum during the measurements. Magnetic susceptibility measurements were performed on a Quantum Design MPMS SQUID susceptometer with a static field of $1 \mathrm{~T}$ in the temperature range of $2-300 \mathrm{~K}$ for the randomly oriented polycrystalline sample $(2.34 \mathrm{mg})$. The sample room was evacuated and backfilled with He gas three times before loading the sample in the magnet. The diamagnetic core contribution was estimated based on Pascal's constants. The value for $(\mathrm{ET})_{3}\left(\mathrm{Br}_{3}\right)_{5}$ thus obtained was $-1.1 \times 10^{-3} \mathrm{emu} \mathrm{mol}^{-1}$. Stability of the $(\mathrm{ET})_{3}\left(\mathrm{Br}_{3}\right)_{5}$ crystal in vacuum was checked by X-ray diffraction of the thin platelet single crystal by comparing the diffraction data obtained before and after keeping it in vacuum for one hour at room temperature. No damage of the crystal by the vacuum treatment was observed. ${ }^{8}$

Diffuse reflectance spectra were measured by a Jasco V-570 in the wavelength range of $350-2300 \mathrm{~nm}$. The solid sample was diluted by $\mathrm{KBr}$, and the measured reflectivity was converted by the Kubelka-Munk function.

\section{Results and Discussion}

Crystal Growth. Although ET is known to form partially oxidized salts when electrolysis is performed with an electrolyte containing $\mathrm{I}_{3}{ }^{-}$or $\mathrm{IBr}_{2}{ }^{-}$, crystal growth with an electrolyte containing $\mathrm{Br}_{3}{ }^{-}$was thought to be difficult. ${ }^{9}$ An exception was found for a solvent system of $\mathrm{C}_{6} \mathrm{H}_{5} \mathrm{COCH}_{3}$. In this solution, poor quality crystals were obtained, but their composition and structure were unknown. In most polar solvents, chemical oxidation of ET by $\mathrm{Br}_{3}{ }^{-}$is thought to occur spontaneously before electrolysis. This has been supposed to prevent the crystal growth of partially oxidized salts of ET with $\mathrm{Br}_{3}{ }^{-}$. However, the true reason may be that it is difficult for $\mathrm{Br}_{3}{ }^{-}$to adopt the same structures and compositions that are seen in the partially oxidized salts of other tri-halides. Since $\mathrm{ET}^{+}$generated by electrolysis is relatively soluble in methanol and is not completely consumed by precipitation of salts with low formal charges, $\mathrm{ET}^{+}$is available for further 
oxidation up to $\mathrm{ET}^{2+}$. Finally, precipitation is believed to occur upon the formation of $(\mathrm{ET})_{3}\left(\mathrm{Br}_{3}\right)_{5}$. When acetonitrile was used instead of methanol as the solvent, only $(\mathrm{ET})_{3} \mathrm{Br}_{2}\left(\mathrm{H}_{2} \mathrm{O}\right)_{2}{ }^{10}$ and $(\mathrm{ET}) \mathrm{Br},{ }^{11}$ which have the $\mathrm{Br}^{-}$counter ion, were obtained.

On the other hand, when the electrolysis of ET was performed using $\mathrm{I}^{-}$instead of $\mathrm{Br}^{-}$in methanol/ $\mathrm{CHCl}_{3}$, only the $\alpha-(\mathrm{ET})_{2} \mathrm{I}_{3}$ salt crystallized. This is thought to result from immediate precipitation of the stable $\alpha-(\mathrm{ET})_{2} \mathrm{I}_{3}$ soon after generation of $\mathrm{ET}^{+}$and $\mathrm{I}_{3}{ }^{-}$in the solution. This means that, even when methanol was included in the solvent system, no salts with high oxidation states were obtained because a sufficient amount of $\mathrm{ET}^{+}$did not persist in the solution. Therefore, the fact that electrolysis of $\mathrm{Br}^{-}$and ET yielded the ET salt with an unusually high oxidation state as a major product suggests that $\mathrm{Br}_{3}{ }^{-}$does not preferably yield partially oxidized ET salts with low oxidation states.

Crystal structure. The crystal structure of $(\mathrm{ET})_{3}\left(\mathrm{Br}_{3}\right)_{5}$ at $123 \mathrm{~K}$ is shown in Fig. 1. The crystal system is monoclinic with space group $P 2_{1} / a, a=14.129(1), b=31.328(3)$, $c=14.536(1) \AA, \beta=106.56(1)^{\circ}$, and $Z=4 .^{7}$ The crystallographically independent units are three whole ET molecules and fifteen $\mathrm{Br}$ atoms. None of the $\mathrm{Br}$ atoms have a positional disorder, and it can be clearly seen that they form five discrete $\mathrm{Br}_{3}$ units; all intra-unit $\mathrm{Br}-\mathrm{Br}$ bond distances are in the range of 2.5-2.6 $\AA$ and all $\mathrm{Br} \cdots \mathrm{Br}$ distances between the $\mathrm{Br}_{3}$ units are longer than $3.8 \AA$. To date, the geometry of the $\mathrm{Br}_{3}{ }^{-}$unit has always been found to be linear (bond angle $=174.8-180^{\circ}$; mean angle $=178.4(14)^{\circ}$ ), and the bond lengths are in the range of $2.41-2.75 \AA$ (mean length $=2.55(6) \AA$ ) (see

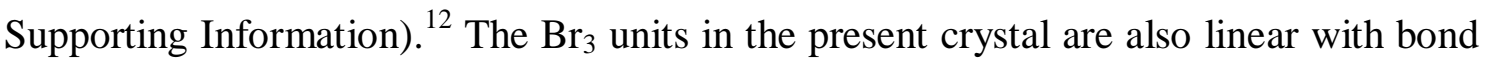
angles of $174.5-179.8^{\circ}$ and bond lengths typical of the $\mathrm{Br}_{3}{ }^{-}$species. Since the thermal parameters of the $\mathrm{Br}$ atoms are all normal with an occupancy of 1 , there is no partial occupation of the sites.

(a)

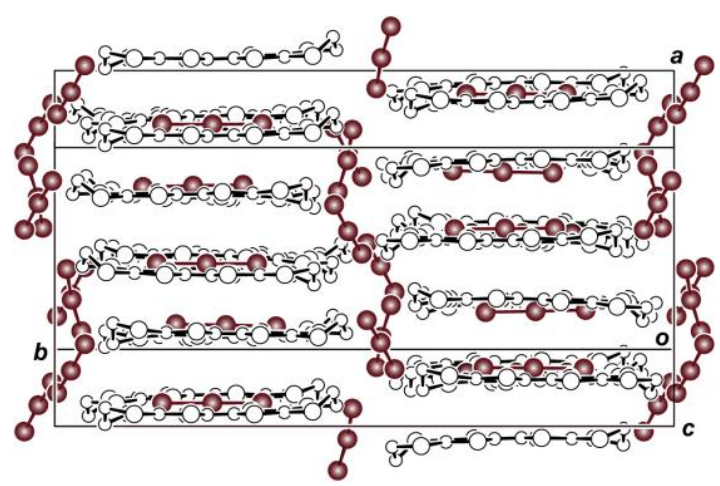


(b)

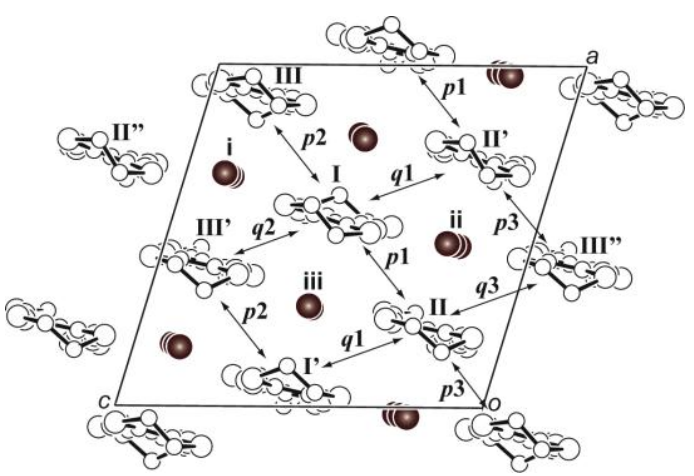

(c)

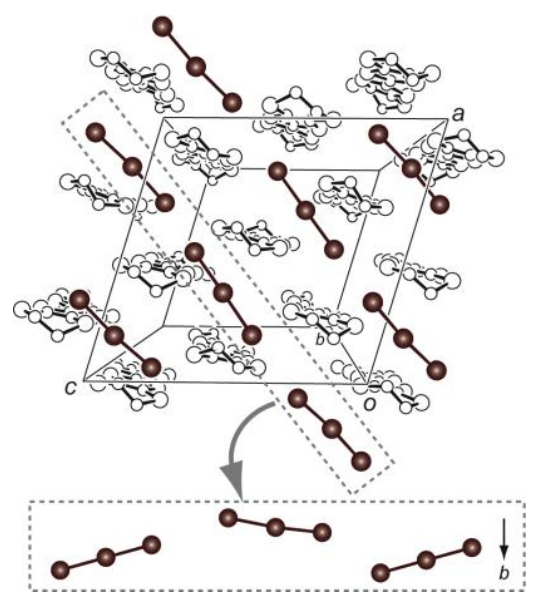

Figure 1. (a) Crystal structure $(123 \mathrm{~K})$ of $(\mathrm{ET})_{3}\left(\mathrm{Br}_{3}\right)_{5}$. (b) $\mathrm{Br}_{3}{ }^{-}$incorporated in ET $2-\mathrm{D}$ sheet (view along the $b$ axis). The HOMO-HOMO overlap integrals $\left(\times 10^{3}\right)$ are $p 1$ : 16.9, p2: 4.7, p3: -18.6, $q 1: 6.5, q 2: 20.1$, and $q 3:-6.0$. (c) $\mathrm{Br}_{3}{ }^{-}$arrangement between the

2-D ET sheets. $\mathrm{Br}_{3}{ }^{-}$in the 2-D sheet is omitted for clarity.

The above results clearly indicate that the formal charge of $\mathrm{ET}_{3} \mathrm{Br}_{15}$ is $\left[\mathrm{ET}^{5 / 3+}\right]_{3}\left[\mathrm{Br}_{3}\right]_{5}$. The bond lengths of ET are known to change based on the formal charge, and two methods for the estimation of charge from the bond lengths have been proposed. $^{13,14}$ The averaged bond lengths and estimated charge $(\gamma)$ of each crystallographically independent ET are summarized in Table 1. The differences between the two methods are rather small, and in both cases, the ET molecules have formal charges larger than one. However, none of the values is as large as +1.67 , which is that expected from the composition. This is considered to be due to inaccuracies in the bond lengths (rather large standard deviations), since the unit cell contains many $\mathrm{Br}$ atoms, which have a larger scattering factor and absorption cross section than those of ET. There also appear to be small charge differences between the ETs. However, when 
taking the bond lengths of $b$ and $c$, which have smaller standard deviations, the differences are found to be rather small.

Table 1. Averaged bond lengths of ET ${ }^{\mathrm{a}}$ and estimated formal charge $(\gamma)$

\begin{tabular}{|c|c|c|c|c|c|c|}
\hline & \multicolumn{4}{|c|}{ bond length / $\AA^{b}$} & \multirow[t]{2}{*}{$\gamma^{\mathrm{c}}$} & \multirow[t]{2}{*}{$\gamma^{\mathrm{d}}$} \\
\hline & $a$ & $b$ & $c$ & $d$ & & \\
\hline ET(I) & $1.364(15)$ & $1.715(10)$ & $1.741(11)$ & $1.389(15)$ & 1.17 & 1.22 \\
\hline ET(II) & $1.395(15)$ & $1.708(10)$ & $1.743(11)$ & $1.391(14)$ & 1.45 & 1.42 \\
\hline ET(III) & $1.369(14)$ & $1.714(10)$ & $1.741(11)$ & $1.387(15)$ & 1.20 & 1.24 \\
\hline
\end{tabular}

${ }^{a}$ Notation is defined in Fig. 1b. ${ }^{b}$ Values in parentheses are the maximum standard deviation. ${ }^{\mathrm{c}}$ Formal charge estimated following ref. 13. ${ }^{\mathrm{d}}$ Formal charge estimated following ref. 14 .

The most noticeable feature of the crystal structure of $(\mathrm{ET})_{3}\left(\mathrm{Br}_{3}\right)_{5}$ is the two-dimensional (2-D) sheet composed of ETs and $\mathrm{Br}_{3}{ }^{-}$anions. This is in contrast to most other partially oxidized ET salts with formal charges less than one, in which the 2-D sheets are generally composed of only ETs. ${ }^{6}$ This is because ETs with high oxidation states can only be assembled when electrostatic attraction to anions is available, as discussed later. Indeed, there are many short $\mathrm{Br} \cdots \mathrm{S}$ contacts and $\mathrm{Br} \cdots \mathrm{ET}$ plane contacts, reflecting strong electrostatic attraction between ET and $\mathrm{Br}_{3}{ }^{-}$. Three $\mathrm{Br}_{3}{ }^{-}$ anions incorporated in the 2-D sheet are surrounded by four ET cations (Fig. 1b; two in the lateral direction and two in the stacking direction), and the shortest contacts along both directions are summarized in Table 2. Considering the van der Waals radii of $\mathrm{Br}$ $(1.85 \AA)$ and $\mathrm{S}(1.80 \AA)$, and the thickness of the aromatic ring $(3.5 \AA),{ }^{15}$ it can be seen that $\mathrm{ET}$ and $\mathrm{Br}_{3}{ }^{-}$are closely located.

Table 2. Shortest contacts between $\mathrm{Br}_{3}{ }^{-}$and $\mathrm{ET}^{\mathrm{a}}$

\begin{tabular}{|c|c|c|}
\hline & $\begin{array}{l}\text { Lateral shortest } \mathrm{Br} \cdots \mathrm{S} \\
\text { contact / } \AA\end{array}$ & $\begin{array}{l}\text { Stacked ET plane }{ }^{\mathrm{b}} \cdots \mathrm{Br} \\
\text { shortest distance / } \AA\end{array}$ \\
\hline $\mathrm{Br}_{3}{ }^{-}(\mathrm{i})$ & $3.345(2)$ & $3.478(4)$ \\
\hline
\end{tabular}




\begin{tabular}{lcccc}
\hline & ET(II") & $3.358(2)$ & ET(III') & $3.419(3)$ \\
\hline $\mathrm{Br}_{3}{ }^{-}$(ii) & ET(I) & $3.398(2)$ & ET(II) & $3.500(2)$ \\
& ET(III") & $3.391(2)$ & ET(II') & $3.543(2)$ \\
\hline $\mathrm{Br}_{3}{ }^{-}$(iii) & ET(II) & $3.329(2)$ & ET(I) & $3.460(2)$ \\
& ET(III') & $3.335(2)$ & ET(I') & $3.438(4)$ \\
\hline
\end{tabular}

${ }^{a}$ Notation is defined in Fig. 1b. ${ }^{b}$ Mean plane calculated using the TTF skeleton.

Aggregation by strong electrostatic attraction between ET and $\mathrm{Br}_{3}{ }^{-}$subsequently induces efficient intermolecular $\pi-\pi$ interaction between the ET cations. The HOMO-HOMO overlap integral values are appreciable in all the neighboring ET pairs. The band structure has 2-D Fermi surfaces with both electron and hole character. ${ }^{7}$

Two other crystallographically independent $\mathrm{Br}_{3}{ }^{-}$anions exist between the $\mathrm{Br}_{3}{ }^{-}$ incorporated 2-D sheets. These anions completely intercept $\pi-\pi$ interactions between the 2-D sheets. Although the $\mathrm{Br}_{3}{ }^{-}$anions are aligned along the [llll $\left.\begin{array}{lll}0 & 1\end{array}\right]$ direction, they do not form an infinite chain but are discrete anions, as shown in Fig 1c. These $\mathrm{Br}_{3}{ }^{-}$anions have short contacts with only ET in the 2-D sheet; most of these are $\mathrm{Br} \cdots \mathrm{H}$ (terminal ethylene group) contacts.

The cohesive force of this crystal is considered to be a sort of ionic bond. Therefore, the lattice is supposed to be harder than those of other partially oxidized salts with formal charges less than one. In order to check this point, the temperature dependences of the lattice parameters were measured. The results are shown in Fig. 2 together with those of $\alpha-(E T)_{2} I_{3}$ as a reference. The volume contraction rate of $(\mathrm{ET})_{3}\left(\mathrm{Br}_{3}\right)_{5}$ is less than one-half that of $\alpha-(\mathrm{ET})_{2} \mathrm{I}_{3}$. This means that the $(\mathrm{ET})_{3}\left(\mathrm{Br}_{3}\right)_{5}$ lattice is more difficult to contract, and indicates that $(\mathrm{ET})_{3}\left(\mathrm{Br}_{3}\right)_{5}$ has features of an ionic crystal. In particular, the temperature dependence of the $b$ axis length is quite small, indicating attractive interactions between $\mathrm{Br}_{3}{ }^{-}$and the terminal ethylene groups of ET in the 2-D sheet dominate the tight packing in $(\mathrm{ET})_{3}\left(\mathrm{Br}_{3}\right)_{5}$. On the other hand, the $\mathrm{Br}_{3}{ }^{-}$incorporated sheet is more shrinkable than along the $b$ axis, reflecting the softer $\pi$-electron clouds of the ET. This tendency is similar with that observed for $\alpha-(E T)_{2} I_{3}$, in which the lattice is harder along the direction perpendicular to the 2-D sheet than over the 2-D sheet. 


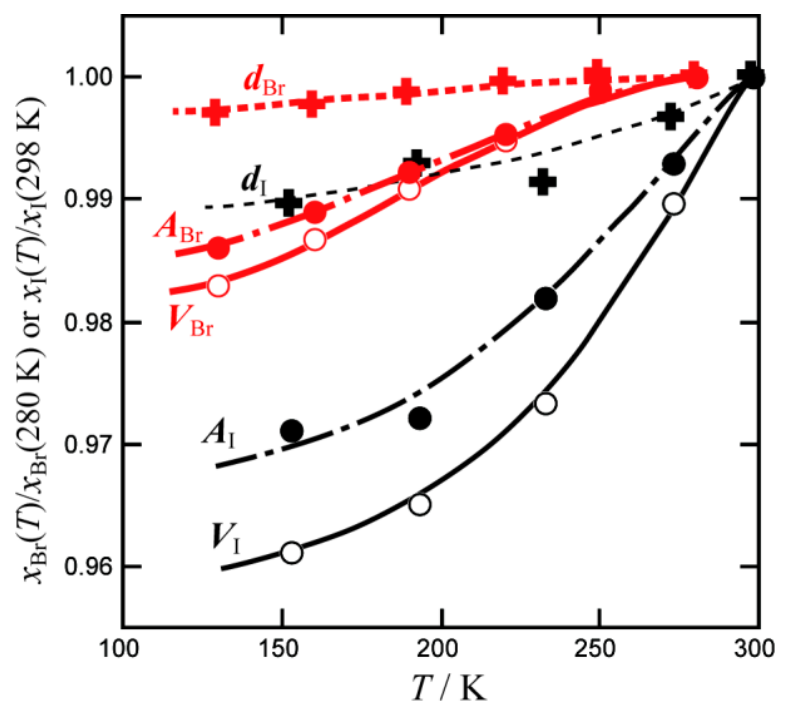

Figure 2. Temperature dependences of the lattice parameters of $(\mathrm{ET})_{3}\left(\mathrm{Br}_{3}\right)_{5}$ (denoted with $\mathrm{Br}$ subscript; normalized at $280 \mathrm{~K}$ ) and $\alpha-(\mathrm{ET})_{2} \mathrm{I}_{3}$ (denoted with $\mathrm{I}$ subscript; normalized at $298 \mathrm{~K}$ ). $V$ : unit cell volume, $A$ : area of the 2-D ET sheet, and $d$ : repeat length along the direction perpendicular to the 2-D sheet. Lines indicate the trends.

Physical properties. In our previous report, ${ }^{7}$ lead wires for resistivity measurements were directly attached to the crystal surfaces by gold or carbon paste. However, it was difficult to obtain reproducible data due to noisy signals below $120 \mathrm{~K}$ probably due to poor contacts at low temperatures. Therefore, we adopted the data with a relatively small $\mathrm{S} / \mathrm{N}$ ratio in the previous report, which showed a slight increase in resistivity below $120 \mathrm{~K}$. In the present study, gold was evaporated onto the crystal surface to reduce the contact resistance. As a result, clear metallic behavior was reproducibly observed below $120 \mathrm{~K}$, as shown in Fig. 3a. However, the resistivity again became scattered at lower temperatures. This is probably due to micro-cracking of the crystal or unstable electromotive force at the interface at low temperatures. Though some anisotropy was observed, it was not conclusive because sample dependence was also observed. In any case, the temperature dependence is metallic along the directions parallel to the 2-D sheet, which is consistent with the band calculation and the temperature dependence of the magnetic susceptibility (Fig. 3b). The Curie tail component at low temperatures is rather small $(0.16 \%)$, and the overall temperature dependence can be considered to be typical Pauli-like paramagnetism. Therefore, it is safe to assume that the metallic state remains at low temperatures. However, the 
susceptibility value is somewhat large for Pauli-like paramagnetism, suggesting that the correlation effect cannot be completely ignored.

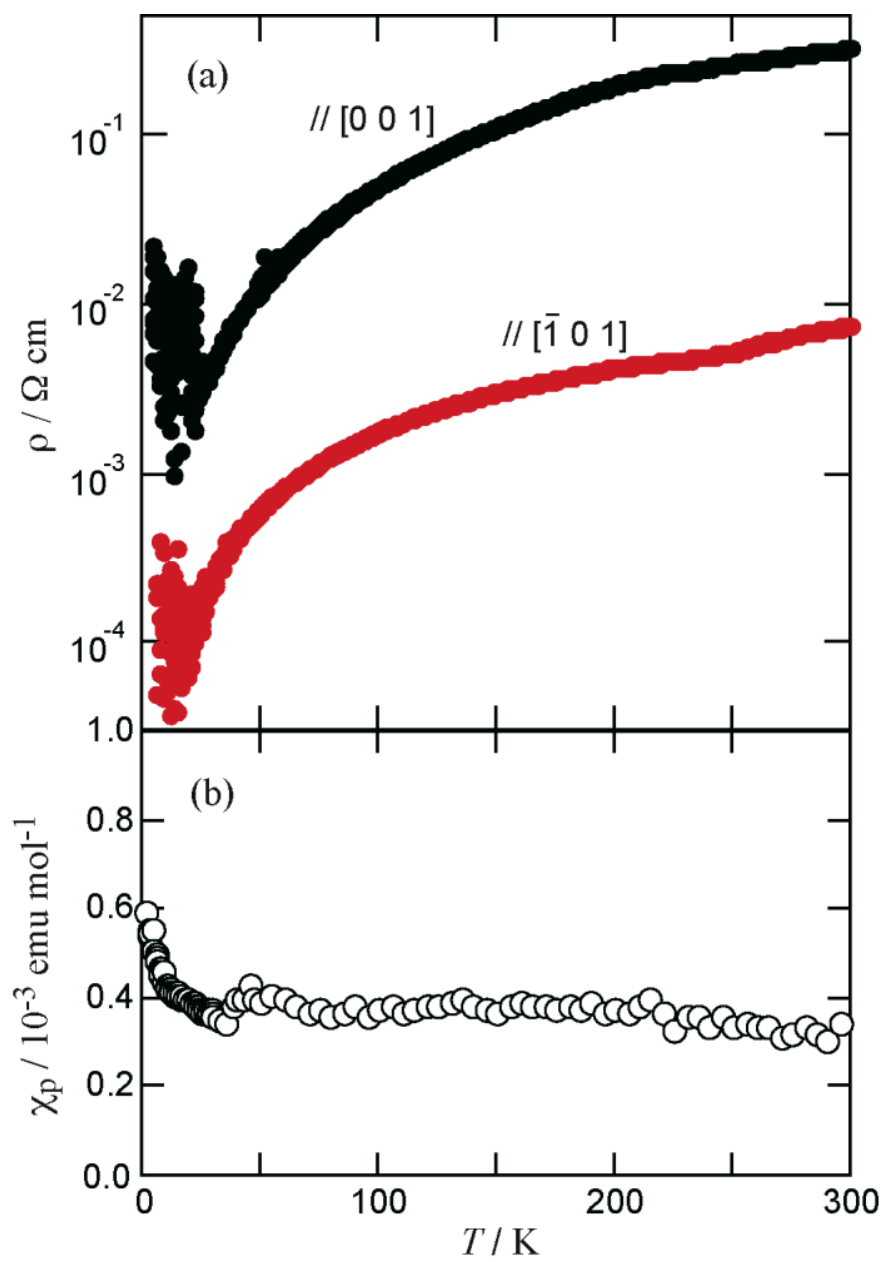

Figure 3. (a) Temperature dependence of the resistivity along the directions parallel to the 2-D sheet in $(\mathrm{ET})_{3}\left(\mathrm{Br}_{3}\right)_{5}$. (b) Temperature dependence of the magnetic susceptibility of $(\mathrm{ET})_{3}\left(\mathrm{Br}_{3}\right)_{5}$.

The optical properties of $(\mathrm{ET})_{3}\left(\mathrm{Br}_{3}\right)_{5}$ in the visible and near-infrared region were examined by the diffuse reflectance method. The Kubelka-Munk function, $f(R)$, which corresponds to the absorption, is shown in Fig. 4. The spectrum of $(\mathrm{ET})_{3}\left(\mathrm{Br}_{3}\right)_{5}$ reveals a characteristic peak at $1.45 \mathrm{eV}$. The intramolecular transition of $\mathrm{ET}^{2+}$ was reported to occur at $855 \mathrm{~nm}(1.45 \mathrm{eV})$ with a shoulder at $714 \mathrm{~nm}(1.74 \mathrm{eV}) .{ }^{16}$ On the other hand, the transition in $\mathrm{ET}^{+}$is known to occur at ca. $1.3 \mathrm{eV}$, as exemplified by $\alpha-(\mathrm{ET})_{2} \mathrm{I}_{3}$ in Fig. 4. The observed peak can be considered to consist of a main contribution from $\mathrm{ET}^{2+}$ and a small contribution from $\mathrm{ET}^{+}$, which is consistent with the formal charge of $\mathrm{ET}^{5 / 3+}$. 


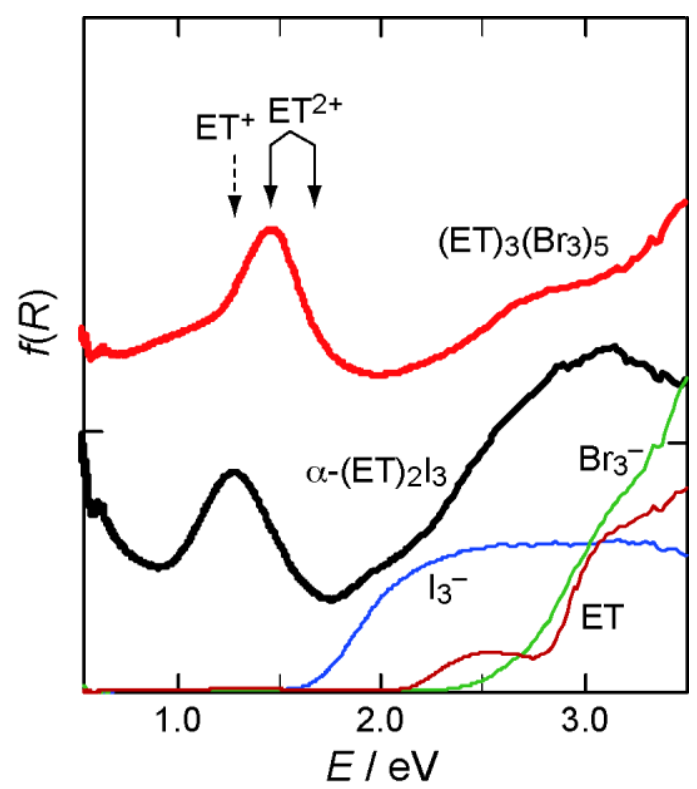

Figure 4. Diffuse reflectance spectra of $(\mathrm{ET})_{3}\left(\mathrm{Br}_{3}\right)_{5}, \alpha-(\mathrm{ET})_{2} \mathrm{I}_{3}, \mathrm{Bu} \mathrm{u}_{4} \mathrm{~N} \cdot \mathrm{Br}_{3}, \mathrm{Bu} \mathrm{u}_{4} \mathrm{~N} \cdot \mathrm{I}_{3}$, and ET. The reflectivity was converted to the Kubelka-Munk function, $f(R)$. The baseline for $(\mathrm{ET})_{3}\left(\mathrm{Br}_{3}\right)_{5}$ is shifted to avoid overlapping.

\section{Structural characteristic of metallic conductors with formal charge exceeding}

one. As mentioned in Introduction, in $\mathrm{C}_{60}$ and some ET salts including the present salt, the components can have formal charges exceeding one. They all have a common feature in that they have charge conduction networks that accommodate counter ions. When the formal charge exceeds one, electrostatic repulsion prevents the molecules from approaching each other, and consequently it is not possible to gain enough transfer energy (Scheme 1a). However, if counter ions are incorporated into the network, electrostatic attraction can operate between the anionic and cationic components and subsequently enable the intermolecular distances between the open-shell molecules to be short enough to gain sufficient transfer energy (Scheme 1b). 
(a)

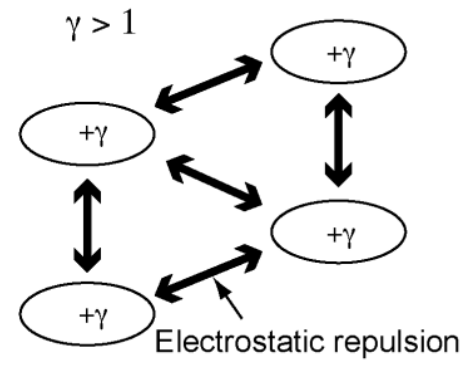

Insufficient $\pi-\pi$ interaction (b)

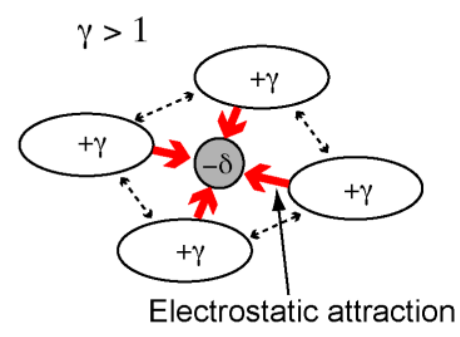

Sufficient $\pi-\pi$ interaction

Scheme 1. Schematic representation of a network of $\pi$-radical cations with a formal charge $(\gamma)$ exceeding one; (a) network without anions and (b) anion-incorporated network.

This structural characteristic is schematically summarized for the ET metallic conductors with formal charges exceeding one and $\mathrm{A}_{3} \mathrm{C}_{60}$ in Fig. 5. In $(\mathrm{ET})_{3}\left(\mathrm{Br}_{3}\right)_{5}$, ET cations and $\mathrm{Br}_{3}{ }^{-}$anions are alternatively arranged along both directions parallel and nearly perpendicular to the ET molecular plane (Fig. 5a). In (ET) ${ }_{6}\left(\mathrm{AuBr}_{2}\right)_{6} \mathrm{Br}(\mathrm{TIE})$, $\mathrm{AuBr}_{2}{ }^{-}$anion is situated in a pocket surrounded by the ETs (Fig. 5b). In the case of $(\mathrm{ET})_{2} \mathrm{Cu}_{3} \mathrm{Br}_{7}\left(\mathrm{H}_{2} \mathrm{O}\right)$, the anion incorporation is inevitable since ET coordinates to the counter metal-complex anion; nevertheless the resultant 2-D sheet has the same feature as those in the other anion incorporated networks of ET (Fig. 5c). Since $\pi-\pi$ interaction between the ETs occurs along various directions, ${ }^{17}$ sufficient $\pi-\pi$ interactions are accomplished even in these ET arrangements. The ( $\pi$-radical cation):(anion) ratio in the 2-D sheet is 1:1 in $(\mathrm{ET})_{3}\left(\mathrm{Br}_{3}\right)_{5}$, whereas it is 3:2 in $(\mathrm{ET})_{6}\left(\mathrm{AuBr}_{2}\right)_{6} \mathrm{Br}(\mathrm{TIE})$ and 2:1 in $(\mathrm{ET})_{2} \mathrm{Cu}_{3} \mathrm{Br}_{7}\left(\mathrm{H}_{2} \mathrm{O}\right)$. Reflecting this ratio, there is no face-to-face or side-by-side approach of ETs in $(\mathrm{ET})_{3}\left(\mathrm{Br}_{3}\right)_{5}$, while such contacts occur in the other two networks with deficient anion contents. Therefore, $(\mathrm{ET})_{3}\left(\mathrm{Br}_{3}\right)_{5}$ is considered to have the strongest ionic-crystal-like feature among these three ET salts. Indeed, only $(\mathrm{ET})_{3}\left(\mathrm{Br}_{3}\right)_{5}$ maintains metallic conductivity down to low temperature among them, which may result from the ionic-crystal-like feature of persistence toward lattice deformation and shrinkage. In $\mathrm{A}_{3} \mathrm{C}_{60}$, the 3-D $\mathrm{C}_{60}$ network incorporates many counter cations (Fig. 5d). The ( $\pi$-anion radical):(cation) ratio is $1: 3$. The sufficient number of counter cation is considered to strengthen the ionic-crystal-like feature. The lattice and its metallic conductivity are stable down to low temperature. 
(a)

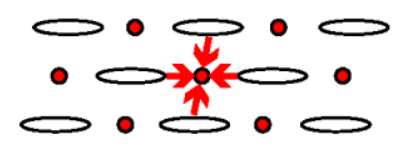

(c)

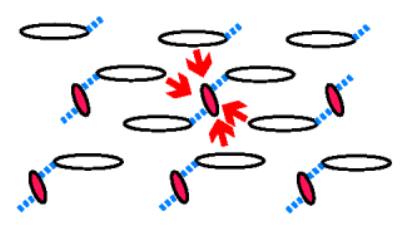

(b)

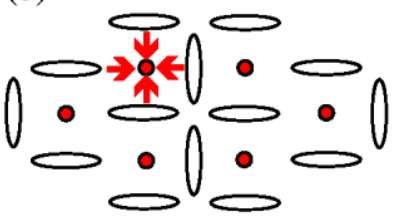

(d)

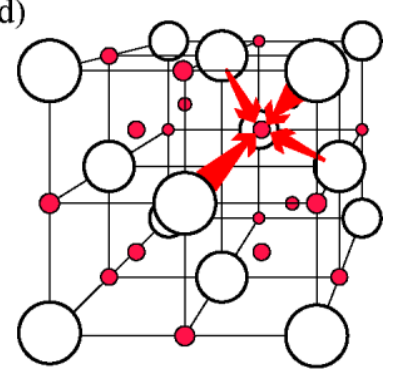

Figure 5. Schematic representation of the counter ion incorporated networks in (a) (ET) $)_{3}\left(\mathrm{Br}_{3}\right)_{5}$, (b) (ET) $)_{6}\left(\mathrm{AuBr}_{2}\right)_{6} \mathrm{Br}$ (TIE), (c) (ET) ${ }_{2} \mathrm{Cu}_{3} \mathrm{Br}_{7}\left(\mathrm{H}_{2} \mathrm{O}\right)$, and (d) $\mathrm{A}_{3} \mathrm{C}_{60}$. 2-D sheets viewed along the long axis of ET in (a), (b), and (c). Anions in the ET sheet are red, while cations are red in (c). Red arrows indicate local electrostatic attraction between $\pi$-radical cations (or anions) and a counter ion. Blue broken lines in (c) indicate coordination bonds.

\section{Conclusion}

The title salt of $(\mathrm{ET})_{3}\left(\mathrm{Br}_{3}\right)_{5}$, in which $\mathrm{ET}^{+1}$ and $\mathrm{ET}^{+2}$ coexist in a mixed valence state, was obtained by electrolysis of ET and $\mathrm{Br}^{-}$in a mixed solvent of methanol/ $/ \mathrm{CHCl}_{3}$. $\mathrm{Br}_{3}{ }^{-}$is incorporated in the 2-D sheet of ET and attractive electrostatic forces between the ET cations and $\mathrm{Br}_{3}{ }^{-}$support aggregation of ET cations with high oxidation states, allowing sufficient $\pi-\pi$ overlap for delocalization of the $\pi$-electrons. The band calculation suggests 2-D metallic character, and the transport properties agree with this interpretation. Electron correlation effects are not directly apparent in these data, and the high oxidation states of the component molecules do not immediately result in specific properties. However, enhancement of the magnetic susceptibility implies that the metallic state might be proximate to some instability such as charge-order states. The ability of this crystal to maintain a stable metallic state at low temperatures may result from its ionic-crystal-like features, in which lattice deformation and/or lattice shrinkage is resisted as compared with typical partially oxidized salts with low oxidation states. This feature is believed to lead to suppression of the phase transitions that accompany 
lattice deformations for energy gain in the electronic system or lattice shrinkage causing enhancement of inter-site correlation effects.

Formation of the anion incorporated 2D sheet is considered to be a common feature for the conducting ET salts with formal charges exceeding one. Availability of $\pi-\pi$ interactions along various directions allows ET to crystallize into metallic conductors with the anion incorporated 2-D sheet structure. Such 2-D sheets have been obtained for ET-type donors even when the oxidation state is less than one. ${ }^{18}$ However, this feature is especially important when the oxidation state exceeds one.

\section{Acknowledgements}

This work was supported in part by Grants-in-Aid for Scientific Research from the Ministry of Education, Culture, Sports, Science and Technology, Government of Japan and Grants-in-Aid for Scientific Research from the Japan Society for the Promotion of Science. The authors thank Profs. Y. Hinatsu and M. Wakeshima at Hokkaido Univ. for their help in the susceptibility measurements.

\section{Supporing Information}

Geometric data of $\mathrm{Br}_{3}^{-}$in various compounds. This material is available free of charge on the web at http://www.csj/journals/bcsj/. 


\section{References}

1 T. Mori, Y. Misaki, T. Yamabe, Bull. Chem. Soc. Jpn. 1997, 70, 1809.

2 Y. Misaki, in TTF Chemistry-Fundamentals and Applications of tetrathiofulvalene, ed. J. Yamada, T. Sugimoto, Kodansya \& Springer, Tokyo, 2004, Chapter 10.

3 R. C. Haddon, Acc. Chem. Res. 1992, 25, 127.

4 a) T. Mori, H. Inokuchi, Y. Misaki, Y. Yamabe, H. Mori, S. Tanaka, Bull. Chem. Soc. Jpn. 1994, 67, 661. b) T. Mori, T. Kawamoto, J. Yamaura, T. Enoki, Y. Misaki, T. Yamabe, H. Mori, S. Tanaka, Phys. Rev. Lett. 1997, 79, 1702.

5 H. M. Yamamoto, J. Yamaura, R. Kato, J. Am. Chem. Soc. 1998, 120, 5905.

6 R. Kanehama, M. Umemiya, F. Iwahori, H. Miyasaka, K. Sugiura, M. Yamashita, Y. Yokochi, H. Ito, S. Kuroda, H. Kishida, H. Okamoto, Inorg. Chem. 2003, 42, 7173.

7 H. Minemawari, T. Naito, T. Inabe, Chem. Lett. 2007, 36, 74.

8 Number of reflections $(I>3 \sigma(I))$ observed for the as-grown crystal was 19685, while that for the same crystal after keeping in vacuum for one hour was 20321 under the same data collection conditions. No meaningful difference in the diffraction data was observed in any $2 \theta$ region.

9 a) H. Anzai, N. Tokumoto, T. Ishiguro, G. Saito, H. Kobayashi, R. Kato, A. Kobayashi, Synth. Met. 1987, 19, 611. b) S. Sakura, H. Imai, H. Anzai, T. Moriya, Bull. Chem. Soc. Jpn. 1988, 61, 3181. c) S. Sakura, H. Anzai, Electrochim. Acta 1993, $38,2343$.

10 H. Urayama, G. Saito, A. Kawamoto, J. Tanaka, Chem. Lett. 1987, 1753.

11 M. A. Beno, G. S. Blackmen, P. C. W. Leung, K. D. Carlson, P. T. Copps, J. M. Williams, Mol. Cryst. Liq. Cryst. 1985, 119, 409.

12 Cambridge Structure Database 2011, Cambridge Crystallographic Data Centre, 12 Union Road, Cambridge, CB2 1EZ, UK.

13P. Guionneau, C. J. Kepert, G. Bravic, D. Chasseau, M. R. Truter, M. Kurmoo, P. Day, Synth. Met. 1997, 86, 1973.

14 G. Saito, H. Izukashi, M. Shibata, K. Yoshida, L. A. Kushch, T. Kondo, H. Yamochi, O. O. Drozdova, K. Matsumoto, M. Kusunoki, K. Sakaguchi, N. Kojima, E. B. Yagubskii, J. Mater. Chem. 2000, 10, 893.

15 A. Bondi, J. Phys. Chem. 1964, 68, 443.

16 L.-K. Chou, M. A. Quijada, M. B. Clevenger, G. F. de Oliveira, K. A. Abboud, D. B. Tanner, D. F. Talharm, Chem. Mater. 1995, 7, 530. 
17 T. Mori, A. Kobayashi, Y. Sasaki, H. Kobayashi, G. Saito, H. Inokuchi, Bull. Chem. Soc. Jpn. 1984, 57, 627.

18 T. Mori, Chem. Rev. 2004, 104, 4947. 
$<$ Graphical Abstracts >

Structural Characteristics in a Stable Metallic ET Salt with Unusually High Oxidation State (ET = bis(ethylenedithio)tetrathiafulvalene)

Hiromi Minemawari, Judy Fe F. Jose, Yukihiro Takahashi, Toshio Naito, and Tamotsu Inabe*

Electrolysis of ET and $\mathrm{Br}^{-}$in methanol/CHCl${ }_{3}$ gave a metallic salt of $(\mathrm{ET})_{3}\left(\mathrm{Br}_{3}\right)_{5}$, in which attractive electrostatic forces between the ET cations and $\mathrm{Br}_{3}{ }^{-}$support aggregation of ET cations with high oxidation states, allowing sufficient $\pi-\pi$ overlap.

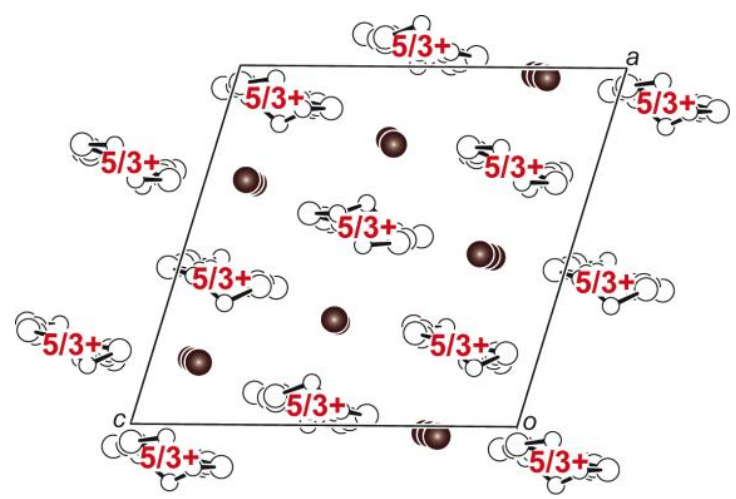

